\title{
Enhancing the Efficiency of moving Video Camera Vigilance using DBSCAN
}

\author{
Ajay Vikram Dev \\ Research Scholar \\ B.N college of Engineering and Technology, Lucknow
}

\begin{abstract}
The author is attempting to build up a model for dynamic or moving camcorder vigilance utilizing Density Based Clustering and area sensors. The authors attempt to exploit the rich usefulness uncovered by the AI worldview in which the stochastic condition to learn is portrayed as a two dimensional diagram where the situation of an object can be given by its directions and coordinates. The author utilizes DBSCAN algorithm alongside sensor empowered test ground zone that keeps the $\mathrm{X}$ and $\mathrm{Y}$ co-ordinates of the moving objects. The approach of the author here is to catch ceaseless video of the densest cluster of objects moving together. One pragmatic use of such framework is a wild scene where gatherings of creatures are moving together to some goal. There will be a to somewhat disorderly aimless movement however we mean to catch just those creatures that are more prominent in number as a gathering and the camera should move imagining them. This can be accomplished by the DBSCAN algorithm.
\end{abstract}

\section{Keywords}

DBSCAN, Unsupervised learning, Sound Navigation and Ranging (SONAR) and laser detection and ranging (LADAR).

\section{INTRODUCTION}

\subsection{Clustering Methods:}

In Clustering the author split the information into groups of comparative objects. Each group is known as a cluster. In comparison of intra-cluster similarity, inter-cluster similarity index is low. It is a significant strategy in data mining. Generally it is viewed as a component of unsupervised learning. Various kinds of clusters as revealed in the literature [1], [2].

\subsubsection{Well Separated Clusters}

Every hub in this sort of cluster is much like each other hub in the cluster, yet not the same as some other hub not in the cluster.

\subsubsection{Centre-Based clusters}

Every article in the group is important to the inside moreover called the Centroid than to the point of convergence of some other cluster.

\subsubsection{Contiguous Clusters}

A center in a cluster is nearest (or even more comparable) to in any event one unique center points in the Cluster when appeared differently in relation to any hub that isn't exist in the cluster.

\subsubsection{Density based clusters}

A cluster is a thick area of points, which is isolated by as per the low-thickness regions, from different locales that is of high thickness.

\subsubsection{Conceptual clusters}

A theoretical cluster transfers some normal element, or demonstrates a specific idea.

\subsection{Use of Clustering and Methods}

Clustering has broad applications in Image Processing, Pattern Recognition, Spatial Data Analysis, Document Classification, Economic Science and Cluster Web log information to find comparative web access designs, and so forth. Different strategies of grouping have been accounted for in literature [3], [4], [5].

\subsubsection{Partitioning method}

In literature different Partitioning methods reported are: $\mathrm{K}$ mean method [3], [4], K-Medoids method (PAM) [5], [6], Farthest First Traversal k-center (FFT) [7], [8], CLARA [9], CLARANS [10], Fuzzy K-Means [11], Fuzzy K-Modes [12], K-Modes [13], Squeezer [14], K-prototypes [15] and COOLCAT [16], etc.

\subsubsection{Hierarchical Methods}

Agglomerative Nesting (AGNES) [17], Divisive Analysis (DIANA) [18], Clustering using Representatives (CURE), Balanced Iterative Reducing and Clustering using Hierarchies $(\mathrm{BIRCH})$ are some of the hierarchical methods.

\subsubsection{Grid Based}

Some of the Grid based clustering methods are STING, Wave Cluster, CLIQUE [19] and MAFIA [20].

\subsubsection{Density Based Methods}

Density based clustering methods include DBSCAN, GDBSCANS, OPTICS, DBCLASD and DENCLUE.

\subsubsection{Model Based method}

Model based methods are divided into two approaches: Statistical approach includes AutoClass method while Neural Network Approach includes Competitive learning and Selforganizing feature maps.

\section{DBSCAN}

(Density Based Spatial Clustering of Applications with Noise) is a well known unsupervised learning technique used in model structure and AI and machine learning algorithms. Before we go any further, we have to characterize what a "unsupervised" learning technique is, Unsupervised learning strategies are when there is no reasonable goal or result we are trying to discover. Rather, we are bunching the data together subject to the equivalence of recognitions.

DBSCAN is a clustering system that is used in machine learning and $\mathrm{AI}$ to dissimilar clusters of high thickness from clusters of low thickness. It is realized that DBSCAN is a density based grouping calculation; it works outstandingly of searching for occupations in the data that have a high thickness of observations, versus areas of the data that are not amazingly thick with recognitions. DBSCAN can sort data into gatherings of changing shapes as well, another strong favored point of view. DBSCAN executes all things considered: 
- Breaks the dataset into $\mathrm{n}$ estimations.

- For each point in the dataset, DBSCAN outlines a $n$ dimensional shape around that data point, and after that checks what number of data centers fall inside that shape.
- DBSCAN thinks about this shape as a cluster. DBSCAN iteratively broadens the bunch, by encountering each individual point inside the group, and checking the amount of other data concentrates close-by. Take the realistic appeared in figure for a model:

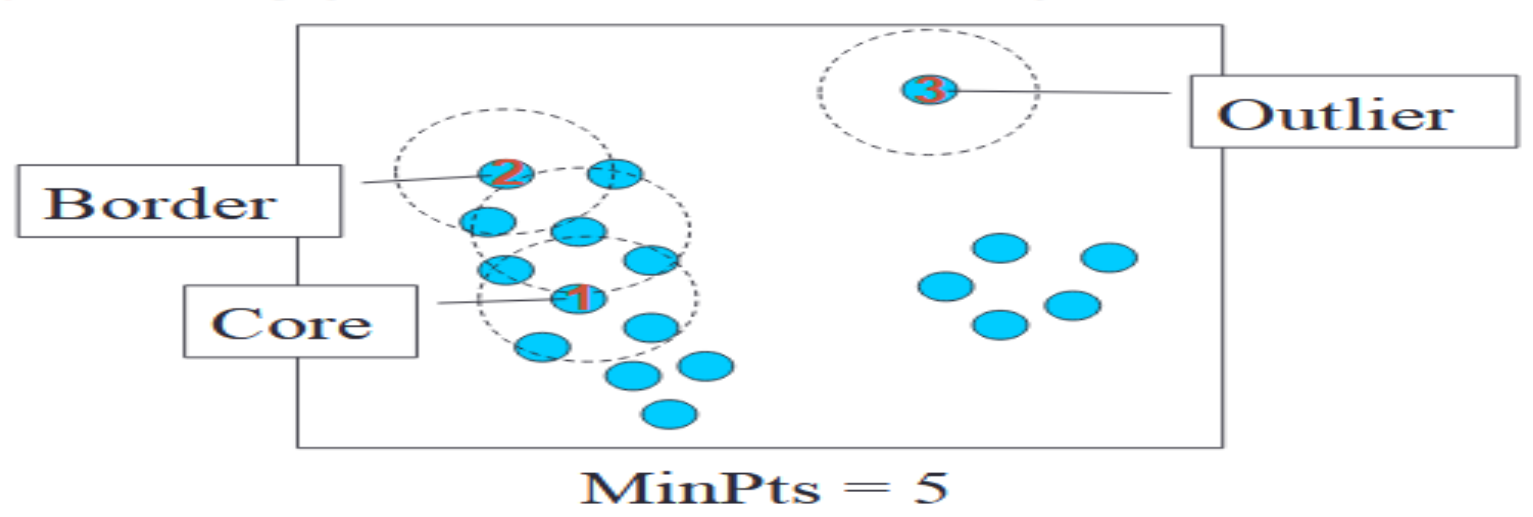

Fig 1: DBSCAN Structur

\section{LOCATION DETECTION AND TRACKING OF MOVING TARGETS}

Many applications require information about an object's location for rescue, emergency and security purposes. The approaches that access an object's location are typically divided into two groups: active and passive localization. In the former approach, the object is associated with a mobile station (MS), such as a tag or device in a communication network. The object's location is determined by sharing data between the MS and the base stations (BSs). The Global Positioning System (GPS), cellular networks, Bluetooth and wireless sensor networks (WSNs) are used in active localization. In the latter approach, the object does not communicate with other devices. However, the object's location can be determined by using the reflected signal from the object. Radio detection and ranging (radar), sound navigation and ranging (sonar) and laser detection and ranging (LADAR) are the most common types of passive localization. These methods have both advantages and disadvantages. However, GPS and long-range radar generate many errors during indoor localization and tracking. Cellular networks and WSNs are limited by their complicated controls and protocols. Sonar and LADAR signals are degraded by environmental interference. Therefore, ultra-wide band (UWB) radar has become an emerging technology that is appropriate for indoor localization and tracking. UWB radar has many advantages, such as a high spatial resolution, the ability to mitigate interference, through-the-wall visibility, a simple transceiver and a low cost.

\section{METHODOLOGY}

The 2 dimensional area, A, assumed square in shape is plotted having $\mathrm{X}$ and $\mathrm{Y}$ coordinates. A random number of moving objects, here assumed to be small robotic cars with constant movement are left in the aforementioned area. Since the area, A, assumed here is small, location tracing sensors are fitted on the boundary of A. A video camera, C, is also planted which is used to position on the selected target. The ideal position of the camera should be on top, middle of A [21].

The DBSCAN algorithm then determines the cluster of robotic cars with maximum density. The algorithm also returns the center of the cluster which is one of the robotic cars. All the cars have built in emitters that generate a specific signal when they are selected as the center of the densest cluster shown in figure 1, called the core. Once the car is selected as the center of the densest cluster, it emits a signal that is received by the location tracing sensors [22]. As soon as the sensors receive the signal, they generate the $\mathrm{X}$ and $\mathrm{Y}$ coordinates of the car that emitted the signal. The coordinates are fed to the camera and the movement of the cluster gets recorded. This process is continuous and if the cluster changes then the process is repeated for the new cluster, center of focus being the new selected center of the densest cluster. The moving camera continuously positions its lens on the moving densest cluster and if the density of the cluster reduces then the new densest cluster is located by the DBSCAN algorithm and the camera starts focusing on the new most densely populated cluster.

The system demonstrated above can also find its application in larger areas. As pointed out earlier that the same process with slight modification can be applied to traffic monitoring and even wild life for framing videos on moving animals in groups, etc. With the aforementioned process a traffic accumulation can be reported or even a traffic jam for the traffic controllers.

In order to find the coordinates in bigger areas, we need the geo-locations in the form of the $\mathrm{X}$ and $\mathrm{Y}$ coordinates of the moving objects and the video camera will be fed with the coordinates as broadcasted by the satellites instead of location tracing sensors [23], [24]. 


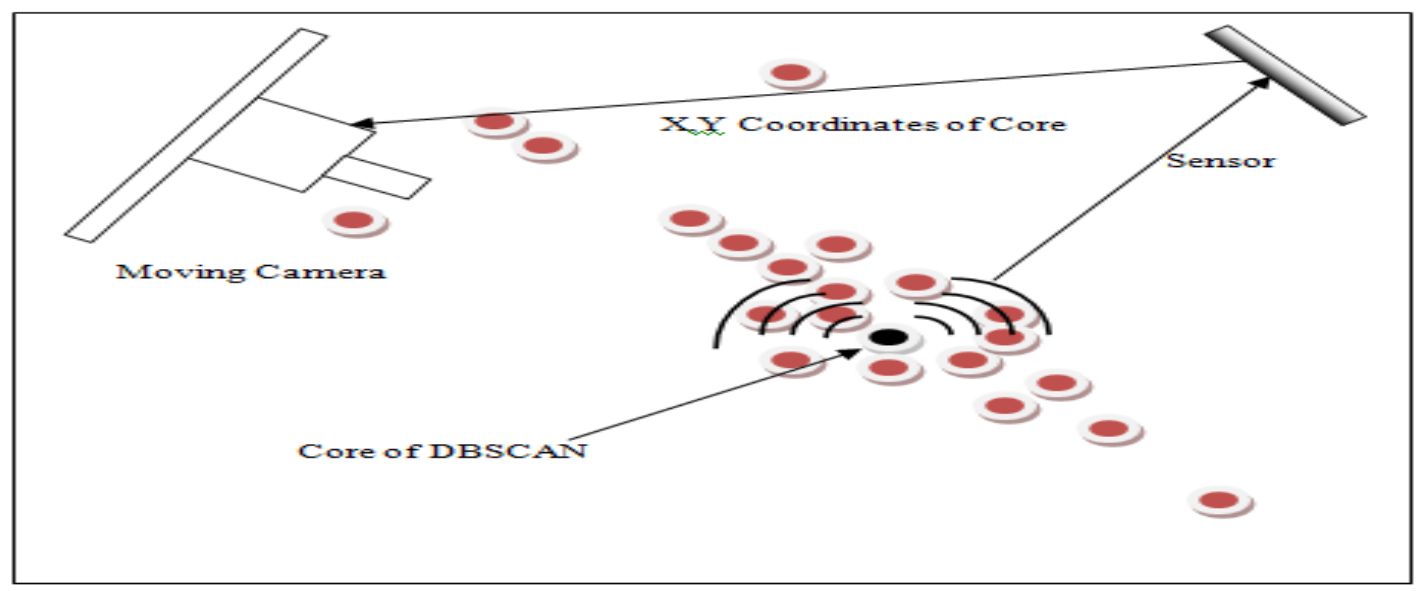

Fig 2: DBSCAN framework

\section{CONCLUSION}

In this paper the author has proposed a model to provide continuous moving camera recording for the most densely populated group of objects. Here, the author has used an unsupervised learning algorithm of the artificial intelligence, called DBSCAN to find out the most intensively crowded orientation of the objects under vigilance. The DBSCAN algorithm reports the densest point, called the core of a population. The crowd is depicted by robotic cars having a facility to emit radio signals. Once a robotic car is selected as the core, it emits radio signals. This signal is received by the sensor installed for this purpose. This sensor calculates the $\mathrm{X}$ and $\mathrm{Y}$ coordinates of the core robotic car and sends them to the positioning system of the camera. With coordinates at hand, the camera focuses its lens on the selected $\mathrm{X}$ and $\mathrm{Y}$ coordinates. In this manner, the automatic moving camera is able to keep track

of the core. With time, the core is changed and so is the camera's focus. It focuses on the new car selected as the core. This installation facilitates a system where the camera always focuses on the densest part of the moving objects. As a future research, this concept can be applied in controlling the traffic, where the radio signals can be replaced by the geo-location finders.

\section{REFERENCES}

[1] K.Kameshwanran, \& Malarvizhi, K. Survey on Clustering Techniques in Data Mining. International Journal of Computer Science and Information Technologies , 5 .2 (2014), 2272-2276.

[2] Junaid, S., \& Bhosle, K. Overview of Clustering Techniques. International Journal of Advanced Research in Computer Science and Software Engineering , 4.11 (2014), 621-624.

[3] Wagstaff, Kiri, et al. "Constrained k-means clustering with background knowledge." Icml. Vol. 1. 2001.

[4] Hartigan, John A., and Manchek A. Wong. "Algorithm AS 136: A k-means clustering algorithm." Journal of the Royal Statistical Society. Series C (Applied Statistics) 28.1 (1979): 100-108.

[5] Park, Hae-Sang, and Chi-Hyuck Jun. "A simple and fast algorithm for K-medoids clustering." Expert systems with applications 36.2 (2009): 3336-3341.

[6] Velmurugan, T., and T. Santhanam. "Computational complexity between $\mathrm{K}$-means and $\mathrm{K}$-medoids clustering algorithms for normal and uniform distributions of data points." Journal of computer science 6.3 (2010): 363.

[7] Panda, Mrutyunjaya, and Manas Ranjan Patra. "A Hybrid clustering approach for network intrusion detection using cobweb and FFT." Journal of Intelligent systems 18.3 (2009): 229-246.

[8] Matsuoka, Hidehiro, and Hiroki Shoki. "Comparison of pre-FFT and post-FFT processing adaptive arrays for OFDM systems in the presence of co-channel interference." 14th IEEE Proceedings on Personal, Indoor and Mobile Radio Communications, 2003. PIMRC 2003.. Vol. 2. IEEE, 2003.

[9] Ng, Raymond T., and Jiawei Han. "E cient and E ective Clustering Methods for Spatial Data Mining." Proceedings of VLDB. 1994.

[10] Ng, Raymond T., and Jiawei Han. "CLARANS: A method for clustering objects for spatial data mining." IEEE Transactions on Knowledge \& Data Engineering 5 (2002): 1003-1016.

[11] Gasch, Audrey P., and Michael B. Eisen. "Exploring the conditional coregulation of yeast gene expression through fuzzy k-means clustering." Genome biology 3.11 (2002): research0059-1.

[12] Huang, Zhexue, and Michael K. Ng. "A fuzzy k-modes algorithm for clustering categorical data." IEEE Transactions on Fuzzy Systems 7.4 (1999): 446-452.

[13] Chaturvedi, Anil, Paul E. Green, and J. Douglas Caroll. "K-modes clustering." Journal of classification 18.1 (2001): 35-55.

[14] He, Zengyou, Xiaofei $\mathrm{Xu}$, and Shengchun Deng. "Squeezer: an efficient algorithm for clustering categorical data." Journal of Computer Science and Technology 17.5 (2002): 611-624.

[15] Huang, Zhexue. "Extensions to the k-means algorithm for clustering large data sets with categorical values." Data mining and knowledge discovery 2.3 (1998): 283304.

[16] Barbará, Daniel, Yi Li, and Julia Couto. "COOLCAT: an entropy-based algorithm for categorical clustering." Proceedings of the eleventh international conference on Information and knowledge management. ACM, 2002.

[17] Struyf, Anja, Mia Hubert, and Peter Rousseeuw. 
"Clustering in an object-oriented environment." Journal of Statistical Software 1.4 (1997): 1-30.

[18] Datta, Susmita, and Somnath Datta. "Comparisons and validation of statistical clustering techniques for microarray gene expression data." Bioinformatics 19.4 (2003): 459-466

[19] De Amorim, Saul G., Jean-Pierre Barthélemy, and Celso C. Ribeiro. "Clustering and clique partitioning: simulated annealing and tabu search approaches." Journal of Classification 9.1 (1992): 17-41.

[20] Goil, Sanjay, Harsha Nagesh, and Alok Choudhary. "MAFIA: Efficient and scalable subspace clustering for very large data sets." Proceedings of the 5th ACM SIGKDD International Conference on Knowledge Discovery and Data Mining. Vol. 443. ACM, 1999.

[21] Ahiska, Yavuz. "Multiple-view processing in wide-angle video camera." U.S. Patent No. 7,450,165. 11 Nov. 2008

[22] Diener, Neil R., David S. Kloper, and Anthony T. Collins. "Server and multiple sensor system for monitoring activity in a shared radio frequency band." U.S. Patent No. 7,184,777. 27 Feb. 2007.

[23] Belisle, Timothy, and Thomas Belisle. "Geo-location system, method and apparatus." U.S. Patent Application No. 11/381,097.

[24] Williams, Darin Scot. "Car-finder method and aparatus." U.S. Patent Application No. 12/157,889.
[25] Pandey. H, and Darbari. M, "Coalescence of Evolutionary Multi-Objective Decision making approach and Genetic Programming for Selection of Software Quality Parameter", International Journal of Applied Information System (IJAIS), Foundation of Computer Science, New York, USA, Volume 7, No. 11, PP. ISSN: 2249-0868, Nov. 2014.

[26] Bansal. S and Pandey. H, "Develop Framework for selecting best Software Development Methodology", International Journal of Scientific and Engineering Research, Volume 5, Issue 4, PP. 1067-1070, ISSN: 2229-5518, Apr. 2014.

[27] Srivastava. M and Pandey. H, "A Literature Review of E- Learning Model Based on Semantic Web Technology", International Journal of Scientific and Engineering Research" Volume 5, Issue 10, PP. 174-178, ISSN: 2229-5518, Oct. 2014.

[28] Pandey. H, “A New NFA Reduction Algorithm for State Minimization Problem”, International Journal of Applied Information Systems (IJAIS), Foundation of Computer Science FCS, New York, USA, Volume 8, No.3, PP. 2730, ISSN: 2249-0868, Feb. 2015.

[29] Pandey. H, "LR Rotation rule for creating Minimal NFA", International Journal of Applied Information Systems (IJAIS), Foundation of Computer Science FCS, New York, USA, Volume 8, No.6, PP. 1-4, ISSN: 2249 0868, Apr. 2015. 